\title{
Análisis del sobrepeso y obesidad, niveles de actividad física y autoestima de la niñez salvadoreña
}

\author{
Evert Iraheta, Boris; Álvarez Bogantes, Carlos \\ Análisis del sobrepeso y obesidad, niveles de actividad física y autoestima de la niñez salvadoreña \\ MHSalud, vol. 17, núm. 1, 2020 \\ Universidad Nacional, Costa Rica \\ Disponible en: http://www.redalyc.org/articulo.oa?id=237061117001 \\ DOI: https://doi.org/10.15359/mhs.17-1.1
}

Esta obra está bajo una Licencia Creative Commons Atribución-NoComercial-SinDerivar 3.0 Internacional. 


\title{
Análisis del sobrepeso y obesidad, niveles de actividad física y autoestima de la niñez salvadoreña
}

\author{
Analysis of Overweight and Obesity, Levels of Physical Activity and Self-Esteem of Salvadoran Children \\ Análise do sobrepeso e obesidade, níveis de atividades físicas e autoestima da infância salvadorenha
}

Boris Evert Iraheta

Universidad de El Salvador, El Salvador

borisevert@gmail.com

(DD http://orcid.org/0000-0001-9243-5391

Carlos Álvarez Bogantes

Universidad Nacional, Costa Rica

ceab.03@gmail.com

(DD http://orcid.org/0000-0003-3797-7993
DOI: https://doi.org/10.15359/mhs.17-1.1

Redalyc: http://www.redalyc.org/articulo.oa? id $=237061117001$

Recepción: 16 Noviembre 2018

Aprobación: 10 Agosto 2019

\section{Resumen:}

El objetivo de este estudio fue determinar el IMC (índice de masa corporal), la autoestima y el nivel de actividad física de los niños y las niñas de II ciclo de la ciudad de San Salvador. Participaron 11 centros escolares: 359 (47 \%) fueron niñas y 399 ( 53 \%) niños, con edad promedio de $11.49 \pm 1.34$ años, una talla de $147.11 \pm 0.10 \mathrm{~cm}$ y un peso de $47.53 \pm 13.758 \mathrm{~kg}$. Se evaluó peso, talla, IMC, la percepción de actividad física y autoestima, por medio del cuestionario de actividad física para niños y niñas mayores (PAQC) y el cuestionario sobre autoestima (LAWSEQ). En los resultados obtenidos se encontró que un $29 \%(\mathrm{n}=227)$ tienen bajo peso, un $46.7 \%(n=354)$ tienen peso saludable, un $18.3 \%(n=139)$ tiene sobrepeso y un $5 \%(n=38)$ tiene obesidad. En el caso de la actividad física los datos recabados muestran que un $53.7 \%(\mathrm{n}=407)$ muestran valores de baja actividad física, un $43.8 \%$ $(n=332)$ valores de actividad física media y un $2.5 \%(n=19)$ valores de actividad física alta. En el caso de la autoestima los datos evidencian que un $52.9 \%(n=401)$ están en condición de baja autoestima, un $43.1 \%(n=327)$ tiene autoestima promedio y un $4.0 \%(n=30)$ presenta valores de alta autoestima. Se concluye que los datos obtenidos en las variables de IMC, nivel de actividad física y autoestima en el grupo de estudiantes evaluados son alarmantes, lo que propone la necesidad de desarrollar estrategias para intervenir esta problemática.

Palabras Clave: sedentarismo, obesidad, estilo de vida sedentario, índice de masa corporal.

\section{AbSTRACT:}

The objective of this study was to determine the BMI (Body Mass Index), the self-esteem and the level of physical activity of the children of the second cycle of the city of San Salvador. 11 schools participated: 359 (47 \%) were girls and 399 (53\%) girls, with an average age of $11.49 \pm 1.34$ years, a height of $147.11 \pm 0.10 \mathrm{~cm}$ and a weight of $47.53 \pm 13.758 \mathrm{Kg}$. Weight, height was evaluated, body mass index, the perception of physical activity and self-esteem, through the Physical Activity Questionnaire for Older Children (PAQ-C), and Lawrence Self-Esteem Questionnaire (LAWSEQ). In the results obtained it was found that 29\% $(n=227)$ are underweight, $46.7 \%(n=354)$ have a healthy weight, $18.3 \%(n=139)$ are overweight and $5 \%(n=38)$ He is obese. In the case of physical activity, the data collected show that $53.7 \%(n=407)$ show values of low physical activity, $43.8 \%(n=332)$ values of physical activity means and $2.5 \%(n=19)$ values of high physical activity. In the case of self-esteem, the data show that $52.9 \%(n=401)$ are in a low self-esteem condition, $43.1 \%(n=327)$ have average self-esteem and $4.0 \%(n=30)$ have high selfesteem values. It is concluded that the data obtained in the variables of body mass index, physical activity level and self-esteem in the students evaluated are alarming, which suggests the need to develop strategies for the intervention of this problem.

KEYWORDS: Sedentary, obesity, sedentary lifestyle, Body mass index.

\section{Resumo:}

O objetivo deste estúdio foi determinar o IMC, a autoestima e o nível de atividade física dos estudantes do segundo ciclo do município de San Salvador. Participaram 11 centros educativos: 359 (47\%) meninas e 399 (53\%) meninos, com uma idade aproximada de $11,49 \pm 1,34$ anos, com $147,11 \pm 0,10 \mathrm{~cm}$ de altura e 47,53 $\pm 13,758 \mathrm{~kg}$ de peso. Foi avaliado peso, altura, índice de massa corporal, percepção de atividade física e autoestima, por meio do questionário de atividade física para crianças e adolescentes 
(PAQ-C) e pesquisa sobre autoestima (LAWSEQ). Nos resultados obtidos, encontrou-se que $29 \%(\mathrm{n}=227)$ estão com baixo peso, $46,7 \%(n=354)$ estão com peso saudável, $18,3 \%(n=139)$ estão com sobrepeso e $5 \%(n=38)$ tem obesidade. No caso da atividade física, os dados recuperados mostram que 53,7\% ( $\mathrm{n}=407)$, tem uma atividade física baixa, 43,8\% $(\mathrm{n}=332)$ tem atividade física média e $2,5 \%(\mathrm{n}=19)$ tem atividade física alta. Sobre a autoestima, os dados deixam em evidencia que 52,9\% ( $\mathrm{n}=401)$ estão em condição de autoestima baixa, 43,1\% $(\mathrm{n}=327)$ têm autoestima regular e 4,0\% $(\mathrm{n}=30)$ apresentam uma alta autoestima. As conclusões indicam que os dados obtidos nas variáveis de índice de massa corporal, nível de atividade física e autoestima dos estudantes avaliados são alarmantes, indicando a necessidade de propor estratégias para a intervenção desta problemática.

Palavras-Chave: sedentarismo, obesidade, estilo de vida sedentário, índice de massa corporal.

\section{INTRODUCCIÓN}

El sobrepeso y la obesidad en la niñez y adolescencia muestran una tendencia al alza en las últimas décadas, la Organización Mundial de la Salud (OMS, 2010) considera que del $4.2 \%$ registrado en 1990 al $6.7 \%$ registrado en el 2010 es razón suficiente para considerar al sobrepeso y la obesidad como una enfermedad global, catalogada como una epidemia. Para el año 2014, la OMS afirma que 41 millones de niños con edad inferior a cinco años padecían de sobrepeso u obesidad, condición que desata una alta probabilidad de ser adultos obesos y desarrollar diabetes, hipertensión u otras enfermedades asociadas (OMS, 2014).

Para el caso de América Latina y el Caribe, la Organización Panamericana de la Salud [OPS] (2017), estima que el $7.2 \%$ de los niños menores de 5 años vive con sobrepeso y obesidad, porcentaje equivalente a 3.9 millones de niños; de los cuales 2.5 millones viven en Sudamérica, 1.1 millones en Centroamérica y 200000 en la región caribeña. Los mayores aumentos en el sobrepeso infantil entre 1990 y 2015 se vieron, en términos de números totales en Mesoamérica, donde la tasa creció de $5.1 \%$ a 7 $\%$, mientras que el mayor aumento en la prevalencia se dio en Caribe, cuya tasa creció de $4.3 \%$ a 6.8 $\%$, mientras que en Sudamérica, la subregión más afectada por el sobrepeso infantil, hubo una disminución marginal y su tasa pasó de $7.5 \%$ a $7.4 \%$ (OPS, 2017).

En El Salvador de acuerdo con los últimos datos oficiales, proporcionados por el Ministerio de Salud de El Salvador [MINSAL], la prevalencia de sobrepeso en niños menores de 5 años es de $6 \%$, en relación con el grupo de edad de 7 a 9 años los valores son el $13 \%$ con sobrepeso y el $10 \%$ con obesidad, dando como resultado un $23 \%$ del total de esta población (MINSAL, 2016). Para el grupo de adolescentes escolares de 13 a 15 años, el $38.4 \%$ presenta problemas de sobrepeso y obesidad, con el $28.8 \%$ y el $9.6 \%$ respectivamente. El problema de sobrepeso y obesidad es más notorio en el sector escolar privado, quienes presentan cifras del $41.2 \%$ y para el sector educativo público, las cifras evidencian un $21.5 \%$ de niños y niñas con sobrepeso y obesidad.

Los datos anteriores vislumbran una situación que se agrava en la edad adulta salvadoreña, específicamente a los 20 años, en donde un $37.6 \%$ presenta sobrepeso y un $26.7 \%$ obesidad, lo que da como resultado un $64.3 \%$ con problemas de sobrepeso y obesidad; situación que vienen a contribuir con otros factores de riesgo para el aumento de enfermedades crónicas no transmisibles (MINSAL, 2016).

Los problemas desarrollados por el sobrepeso y la obesidad en la niñez y adolescencia son diversos, en la dimensión psicológica se presenta problemas de autoestima, familiares, interpersonales y hasta académicos. En la orgánica desata patologías lipídicas, problemas de la piel, desbalance vitamínicos y hormonales, entre otros. Y en la económica, el costo para suplir las necesidades de los niños obesos es cada vez mayor para los sistemas de salud pública (Aparco, Bautista-Olórtegui, Astete-Robilliard y Pillaca, 2016).

Ambas condiciones se desarrollan debido a dos razones fundamentales, la asimetría energética de las calorías consumidas y las gastadas; y el descenso de la actividad física como producto de las nuevas configuraciones sociales. En el ámbito mundial, el $23 \%$ de los adultos y el $81 \%$ de los adolescentes en edad escolar no mantienen suficiente actividad (OMS, 2010), a lo que se le agrega el consumo de alimentos hipercalóricos y da como resultado un aumento en el peso corporal. 
El asunto de la inactividad física es inherente a todos los países, la nueva cosmovisión modifica el comportamiento de las personas. La transición al postmodernismo cada día arrecia el sedentarismo y limita las posibilidades de movimientos, esto es evidente en la niñez y la adolescencia, donde han sustituidos los juegos que implica movimientos, por horas de pantalla, tiempo dedicado a teléfonos inteligentes, tabletas, televisiones y computadoras, condición que lleva al aumento del sobrepeso y la obesidad, problemas relacionados con la atención, ansiedad, autoestima, depresión y alteraciones del sueño (Gahagan, 2016).

La inactividad física es un fenómeno global que se extiende a un ritmo acelerado por todo el mundo, la OMS (2010), expone que un 60 \% de la población mundial no realiza la actividad física necesaria para obtener beneficios en la salud, situación por la cual es considerada el cuarto factor de riesgo de mortalidad, que causa la muerte de 32 millones de personas cada año. Y es el principal causante del padecimiento de las Enfermedades No Transmisibles (ETN), las cuales evolucionan lentamente y son responsables de los altos costos de los sistemas de salud pública (García y González-Jurado, 2017).

La práctica regular de actividad física reduce el riesgo de padecer hipertensión arterial, cardiopatías coronarias, accidentes cerebro vasculares, diabetes, cáncer de mama y colon; además mejora la salud ósea y contribuye para aumentar el gasto energético, aspecto fundamental asociado al equilibrio calórico y control adecuado del peso corporal; no obstante la OMS (2010), estima que más del $80 \%$ de la población adolescente del mundo no tiene un nivel suficiente de actividad física, lo que implicaría un alto riesgo de padecer las afecciones antes descritas.

En El Salvador se estima que 1059469 personas padecen obesidad; 1051424 padecen dislipidemias; 487 875 diabetes mellitus; y 1446381 padecen hipertensión arterial (MINSAL, 2015). Todos estos productos de la inactividad física, si bien es cierto en el ámbito nacional no se cuenta con estudios relacionados con la medición del nivel de actividad física, las cifras ponen en evidencia que hay una población con un bajo nivel de actividad física y alto sedentarismo.

Por otro lado, la autoestima es una construcción, que parte de las primeras nociones que un individuo tiene sobre sí mismo, estas nociones son aprendidas en el núcleo familiar y se desarrollan a lo largo de la vida lo que indica que no es estable en el tiempo, por tanto, puede ser modificada; una autoestima saludable se relaciona con el desarrollo integral de la personalidad (Araujo, Cerritos y Molina, 2017). Estudios como los de Zamani et al., (2016) reportan que los altos niveles de sobrepeso y obesidad y los niveles de sedentarismo, podrían dar como resultado una disminución de la percepción de la autoeficacia, la competencia física y la aceptación social, lo que podría generar cambios evidentes en la autoestima en la niñez. Por consiguiente, es importante que esta mantenga valores altos en lo jóvenes, dado que es un factor asociado a las adaptaciones psicosociales y los niveles bajos de esta crean una serie de síntomas psicopatológicos como: ansiedad, estados de ánimo bajo, consumo de sustancias psicoactivas, agresiones hacia sí mismo y hacia los demás, entre otros, ya que una persona que no ha construido amor propio difícilmente establece relaciones saludables con sus iguales y tendrá dificultades en el desarrollo y expresión de sus afectos (Araujo, Cerritos y Molina, 2017).

Asimismo, la exposición a la violencia deteriorara el desarrollo de la personalidad de los niños y niñas; en El Salvador existen niveles altos de ella, para el año 2014 el Instituto Universitario de Opinión pública de la UCA [IUDOP] (2014), estimaba que, a 23 años de la firma de los acuerdos de paz, se habían contabilizado setenta y tres mil muertes, cifra similar al periodo de la guerra civil, ahora en posguerra. A lo que se le agrega la violencia intrafamiliar, la que, debido a los arquetipos sociales de la población salvadoreña, crea condiciones para deteriorar la autoestima ya que las relaciones de poder, la asignación de roles y el modelo crianza con estructuras rígidas utilizan el castigo físico, las amenazas y el irrespeto a los derechos humanos de los niños y niñas, como un método correctivo, situación que tiene serias repercusiones en la autoestima para lograr un pleno desarrollo (Manzano, 2016).

Lo antes descrito no permite crear ambientes adecuados para fortalecer la personalidad de los niños y las niñas, ya que los entornos violentos, la carencia de afecto, el abandono de los padres, el maltrato físico y psicológico, son elementos que ocasionan anormalidades en el desarrollo emocional y esto, como 
consecuencia, puede llevar al consumo de sustancias psicoactivas, enfermedades psicosomáticas, alteraciones de la personalidad y conductas negativas respectos a las normas sociales (Araujo, Ceritos y Molina, 2017).

Por tanto, el desarrollo de este estudio es importante, ya que ayudará a crear un modelo de evaluación que permita conocer el estado de sobrepeso y obesidad, nivel de actividad física y autoestima de los niños, niñas y adolescentes salvadoreños, el que aspira a crear inferencia en las políticas públicas nacionales y del istmo centroamericano, con la finalidad mejorar la calidad de vida de los niños, niñas y adolescentes.

Consecuentemente, el propósito de este estudio fue analizar la prevalencia del sobrepeso u obesidad, los niveles de actividad física y de autoestima de los estudiantes de cuarto a sexto de educación básica de diez escuelas del municipio de San Salvador.

\section{Metodología}

\section{Tipo de estudio}

El desarrollo de estudio fue de carácter transversal descriptivo-inferencial, se tomó una muestra por conglomerados (Hernández-Sampieri, Fernández-Collado y Baptista, 2010) de los siete distritos escolares del municipio de San Salvador, El Salvador, en donde las autoridades de la departamental de educación, directivos escolares y padres dieron autorización a participar

\section{Participantes}

Los sujetos de estudio fueron escolares de cuarto a sexto grado, en edades de 9 a 13 años, esta población estaba compuesta por 399 del sexo masculino y 359 del sexo femenino, para dar un total de 758. El procedimiento para la obtención de la muestra fue probabilística aleatoria por conglomerados, en donde se realizó una selección estratificada del número de centros escolares del municipio de San Salvador, con el requisito que debían ser públicas, poseer segundo ciclo de educación básica, y tener una matrícula igual o mayor a un centenar de estudiantes.

\section{Instrumentos}

Las variables analizadas en esta investigación fueron el Índice de Masa Corporal, Nivel de Actividad Física y Autoestima.

\section{Indice de masa corporal}

Se analizó a través de las mediciones de la talla, y el peso corporal, las cuales fueron medidas a través del estadiómetro marca SECA 213 y la báscula marca TANITA UM-08, y se utilizó la Calculadora del percentil del IMC en niños y adolescentes versión métrica del Centro de Control y Prevención de Enfermedades (CDC, 2015), disponible en: https://www.cdc.gov/, para el cálculo de índice de masa corporal de acuerdo los cuadros de crecimiento por percentil de edad establecidos, a partir de la fecha de nacimiento, el día de la medición, el peso y la talla de la niñez. 


\section{Nivel de actividad física}

Se midió con el Cuestionario de actividad física para niños y niñas mayores (PAQ-C), validado al español por Martínez-Gómez, et al. (2009). Este es un cuestionario autoadministrado diseñado para medir actividad física moderada a vigorosa en niños y adolescentes entre los 8 y los 14 años, realizada en los últimos 7 días, consiste en diez ítems, nueve de los cuales se utilizan para calcular el nivel de actividad y el otro evalúa si alguna enfermedad $u$ otro acontecimiento impidieron que el niño hiciera sus actividades regulares en la última semana. El resultado global del test es una puntuación de 1 a 5 , de tal forma que las puntuaciones más altas indican un mayor nivel de actividad (Crocker, Bailey, Faulkner, Kowalski y McGrath, 1997). Asimismo, cuenta con una adecuada confiabilidad test-retest $(\mathrm{r}=0.75$ a 0.82$)$ y una validez razonable $(\mathrm{r}=0.45$ a 0.53$)$. Conscientes de las diferencias culturales, este cuestionario fue sometido a una valoración lingüística, para poder ser aplicado en El Salvador.

\section{Autoestima}

Se valoró con el cuestionario LAWSEQ, adaptado al español por Marcó y de Gracia (2000), el cual se fundamenta en una escala que valora la autoestima en niños niñas y se basa en la propuesta de Lawrence (1981), quien propone que la autoestima es una evaluación afectiva que los niños y las niñas hacen de la suma de sus características físicas y cognitivas, está compuesto por 16 reactivos con 3 posibles respuestas, "sí", "no" y "no lo sé”. Reporta una fiabilidad interna de $\alpha=0.76$ (Lawrence, 1981) y una validez externa de ( $\mathrm{r}=0.73$ ), con respecto al Coopersmith Self-Esteem Inventory.

\section{Procedimiento}

Las autoridades de la departamental de educación y directivos escolares autorizaron la realización del estudio. Los padres de familia o encargados de la custodia legal de los escolares fueron informados del desarrollo y el objetivo del estudio y firmaron un documento de consentimiento informado. Después de obtener los permisos legales, consentimientos informados y el asentimiento de los participantes, se dispuso a realizar la recolección de los datos entre los meses de septiembre a noviembre de 2017. En la primera visita se centró en dar conocer el proyecto al director del Centro Escolar e informar a los docentes responsables de cada sección. En la segunda visita se realizaron principalmente la aplicación de los cuestionarios (PAQ-C y LAWSEQ) y una vez completados se realizaron las mediciones de talla y peso, según los procesos protocolarios. Todas las mediciones se realizaron en un día regular de clases y en estricta concordancia con los principios éticos para la investigación indicados en la Declaración de Helsinki.

\section{Análisis estadísticos}

Los datos se tabularon y analizaron, a través del programa informático estadístico SPSS 15.0 para Windows. Se hizo un análisis estadístico inicial de normalidad. Se realizaron estudios de corte descriptivo (medias y desviaciones estándar y frecuencias) con todas las variables estudiadas: sexo, edad, nivel educativo, talla, peso, valoración de IMC, nivel de actividad física y valoración de autoestima, y de las frecuencias para descartar valores atípicos. En las pruebas inferenciales de ANOVA factorial $2 \times 3$ para determinar las diferencias entre sexo y grado escolar se utilizó un criterio de significancia de $\mathrm{p}<0.05$. 
MHSALUD, ISSN: 1659-097X, 17(1), ENERO-JuNIO, 2020, PP 1-15

Evert Iraheta, Álvarez Bogantes

\section{Resultados}

En la Tabla 1 se observa la descripción de las variables estudiadas.

TABLA 1

Promedios y desviaciones estándar de las variables del estudio

\begin{tabular}{lccccc}
\hline Variables & $\mathbf{n}$ & Media & $\begin{array}{c}\text { Desviación } \\
\text { estándar }\end{array}$ & Mínimo & Máximo \\
\hline Edad & 758 & 11.49 & 1.34 & 9 & 16 \\
Talla (cm) & 758 & 147.11 & 0.10 & 1.23 & 1.96 \\
Peso $(\mathbf{k g})$ & 758 & 47.53 & 13.78 & 24 & 99 \\
IMC (km/m²) & 758 & 21.62 & 4.65 & 9.10 & 35.70 \\
PAQ-C & 758 & 2.95 & 0.84 & 0.8 & 4.9 \\
LAWSEQ & 758 & 14.45 & 5.11 & 0 & 24 \\
\hline
\end{tabular}

Nota: IMC=Índice de masa corporal; PAQ-C=Mediciones de nivel de actividad física; LAWSEQ=Mediciones del nivel de autoestima

Los datos obtenidos muestran que un $23.3 \%$ (177) de la muestra, están en condición de sobre peso y obesidad, un $46.7 \%$ (354) están con peso normal y un $29.9 \%$ (227) se encuentra con bajo peso. Con respecto al IMC, se aprecia que este tiende a aumentar junto con la edad, tanto para masculino como para femenino. En relación con el nivel de actividad física (PAQ-C), los valores evidencian que a mayor edad menor nivel de actividad física, puesto que los promedios van disminuyendo para niñas y niños. Para el caso de la autoestima (LAWSEQ) un $52.90 \%$ tiene baja autoestima, un $43.14 \%$ están con autoestima promedio y un $3.96 \%$ presentan alta autoestima, se observa que hay una tendencia a mantener el promedio de autoestima tanto para niños y niñas (ver Tabla 2). 
TABLA 2

Comparación de las variables índice de masa corporal, nivel de actividad física y autoestima según sexo y edad

\begin{tabular}{|c|c|c|c|c|c|}
\hline Sexo & $\begin{array}{l}\text { Edad } \\
\text { (años) }\end{array}$ & n & $\begin{array}{c}\mathrm{IMC} \\
\left(\mathrm{kg} / \mathrm{m}^{2}\right) \\
\end{array}$ & $\begin{array}{c}\text { PAQ-C } \\
\text { (Actividad física) } \\
\end{array}$ & $\begin{array}{c}\text { LAWSEQ } \\
\text { (Autoestima) }\end{array}$ \\
\hline \multirow{5}{*}{ Niños } & 9 & 23 & $19.15 \pm 4.27$ & $3.00 \pm 0.89$ & $1.43 \pm 0.51$ \\
\hline & 10 & 87 & $20.65 \pm 4.65$ & $3.18 \pm 0.84$ & $1.46 \pm 0.57$ \\
\hline & 11 & 98 & $20.90 \pm 4.11$ & $2.76 \pm 0.81$ & $1.62 \pm 0.58$ \\
\hline & 12 & 116 & $22.23 \pm 4.55$ & $2.89 \pm 0.89$ & $1.49 \pm 0.55$ \\
\hline & 13 o más & 75 & $24.33 \pm 4.74$ & $2.75 \pm 0.82$ & $1.32 \pm 0.50$ \\
\hline \multirow{5}{*}{ Niñas } & 9 & 22 & $19.71 \pm 4.39$ & $3.21 \pm 0.83$ & $1.55 \pm 0.51$ \\
\hline & 10 & 61 & $20.39 \pm 4.39$ & $3.22 \pm 0.84$ & $1.48 \pm 0.59$ \\
\hline & 11 & 82 & $20.45 \pm 4.07$ & $3.06 \pm 0.90$ & $1.55 \pm 0.61$ \\
\hline & 12 & 113 & $21.86 \pm 4.60$ & $2.96 \pm 0.78$ & $1.58 \pm 0.62$ \\
\hline & 13 o más & 81 & $23.17 \pm 4.74$ & $2.80 \pm 0.74$ & $1.53 \pm 0.55$ \\
\hline$p$ & & & 0.001 & 0.001 & 0.33 \\
\hline
\end{tabular}

Nota: IMC=Índice de masa corporal; PAQ-C=Mediciones de nivel de actividad física; LAWSEQ=Mediciones del nivel de autoestima.

En la Tabla 2 se observa, que al comparar los datos del estudiantado de manera general, los promedios del IMC por sexo son similares, no obstante presenta un leve aumento en los niños (21.78), respecto a las niñas (21.45), al analizarlos esta misma variable por edad se encuentran diferencias significativas en las edades de 12 y 13 años $(F(9)=3.28 ; p=0.001)$, donde hay un incremento de los promedios en ambos sexos. Caso similar se observa en la variable nivel de actividad física (PAQ C), ya que las niñas y los niños están en un nivel moderado, pero el promedio de las niñas es mayor (3.00) en relación al de los niños pues presenta un valor disminuido (2.90), en referencia a las edades, los niños y niñas de 11, 12 y 13 años tienden a disminuir su nivel de actividad física en ellas $(\mathrm{F}(9)=7.48 ; \mathrm{p}=0.001)$. También, al comparar los datos de la variable autoestima (LAWSEQ), se presentan valores promedios para las niñas con un leve aumento (1.63) comparado al promedio de los niños (1.51) pero no se encontraron diferencias significativas por edad $(\mathrm{F}(9)=1.14 ; \mathrm{p}=0.33)$.

Al contrastar los resultados por grado escolar (ver Tabla 3), se puede observar que hay diferencias significativas en el índice de masa corporal en $4^{\circ}, 5^{\circ}$ y $6^{\circ}$, en las edades de 11,12 y 13 años donde se muestra una tendencia al aumento en esta variable $(F(13)=5.85 ; \mathrm{p}=0.001)$. En cuanto al nivel de actividad física, hay diferencias significativas en $4^{\circ}$ donde se presenta un nivel moderado de actividad física en la mayoría de edades $(\mathrm{F}(13)=2.48 ; \mathrm{p}=0.002)$. Por otro lado, al analizar los resultados por edades, se observa una tendencia a 
MHSALUD, ISSN: 1659-097X, 17(1), ENERo-Junio, 2020, PP 1-15

Evert Iraheta, Álvarez Bogantes

disminuir la práctica de actividad física entre los 11, 12 y 13 años de edad. En la autoestima, no se presentaron diferencias ni por grado escolar ni edad $(F(13)=1.19 ; \mathrm{p}=0.27)$, sin embargo se observa una disposición a disminuir el nivel de autoestima conforme se acercan a la etapa de la pubertad.

TABLA 3.

Comparación de las variables índice de masa corporal, nivel de actividad física y autoestima según la edad y el grado escolar

\begin{tabular}{|c|c|c|c|c|c|}
\hline Variables & $\begin{array}{l}\text { Edad } \\
\text { (años) }\end{array}$ & $\mathbf{n}$ & $\begin{array}{c}\text { IMC } \\
\left(\mathrm{kg} / \mathrm{m}^{2}\right)\end{array}$ & $\begin{array}{c}\text { PAQ-C } \\
\text { (Actividad } \\
\text { fisica) }\end{array}$ & $\begin{array}{c}\text { LAWSEQ } \\
\text { (Autoestima) }\end{array}$ \\
\hline \multirow{5}{*}{ Cuarto } & 9 & 44 & $19.50 \pm 4.30$ & $3.09 \pm 0.86$ & $14.22 \pm 4.29$ \\
\hline & 10 & 138 & $20.42 \pm 4.46$ & $3.18 \pm 0.85$ & $14.70 \pm 4.87$ \\
\hline & 11 & 46 & $21.38 \pm 4.50$ & $3.04 \pm 0.94$ & $14.95 \pm 5.58$ \\
\hline & 12 & 13 & $22.00 \pm 4.09$ & $3.28 \pm 0.50$ & $13.07 \pm 6.87$ \\
\hline & 13 o más & 7 & $21.88 \pm 4.46$ & $2.54 \pm 0.89$ & $11.85 \pm 5.92$ \\
\hline \multirow{5}{*}{ Quinto } & 9 & 1 & $16.10 \pm 0.00$ & $3.20 \pm 0.00$ & $16.00 \pm 0.00$ \\
\hline & 10 & 9 & $21.27 \pm 4.65$ & $3.37 \pm 0.47$ & $12.66 \pm 4.27$ \\
\hline & 11 & 11 & $20.72 \pm 3.92$ & $2.79 \pm 0.80$ & $14.72 \pm 4.39$ \\
\hline & 12 & 78 & $21.86 \pm 4.39$ & $2.80 \pm 0.93$ & $14.30 \pm 5.56$ \\
\hline & 13 o más & 25 & $24.33 \pm 5.04$ & $2.87 \pm 0.65$ & $12.80 \pm 4.83$ \\
\hline \multirow{4}{*}{ Sexto } & 10 & 1 & $31.00 \pm 0.00$ & $2.80 \pm 0.00$ & $6.00 \pm 0.00$ \\
\hline & 11 & 23 & $19.14 \pm 3.71$ & $3.09 \pm 0.91$ & $16.73 \pm 4.95$ \\
\hline & 12 & 138 & $22.15 \pm 4.74$ & $2.95 \pm 0.78$ & $14.54 \pm 5.67$ \\
\hline & 13 o más & 124 & $23.70 \pm 4.68$ & $2.77 \pm 0.79$ & $14.19 \pm 4.95$ \\
\hline$p$ & & & 0.001 & 0.002 & 0.27 \\
\hline
\end{tabular}

Nota: IMC=Índice de masa corporal; PAQ-C=Mediciones de nivel de actividad física; LAWSEQ=Mediciones del nivel de autoestima

\section{Discusión}

El propósito de esta investigación fue analizar la prevalencia del sobrepeso y obesidad, el nivel de actividad física y de autoestima de los estudiantes de cuarto a sexto grado de educación básica en diez escuelas del municipio de San Salvador. Los resultados de esta investigación en la variable de peso corporal mostraron que 
$23 \%$ de los evaluados presentan sobrepeso y obesidad, lo que expone a esta población a sufrir enfermedades cardiovasculares y diabetes (Buchan, Ollis, Thomas y Baker, 2012).

En consonancia con lo expresado por la OMS (2016) el sistema de salud salvadoreño enfrenta una epidemia, que requiere de la atención inmediata. Evidentemente los resultados de este estudio son alarmantes y adquieren mayor realce cuando los comparamos con el IV Censo Nacional de talla y I Censo de Nacional de peso de El Salvador en escolares de primer grado realizado por CONASAN (2016), donde se reportaron datos de sobrepeso y obesidad del $30.74 \%$, condición que vislumbra una prevalencia de sobrepeso y obesidad a medida que avanzan en edad.

Estudios Centroamericanos han mostrado resultados similares en esta variable. Por ejemplo: en Nicaragua en el 2017, se reportaron niveles de sobrepeso de 25 \% (Reyes, Otero, Pastrán, Herrera-Monge y Álvarez, 2018). Adicionalmente, un estudio realizado en Guatemala arrojó porcentajes del $40 \%$ de sobrepeso y obesidad de los estudiantes evaluados en 2017 (Dubon y Aguilar, 2018).

Los estudios citados anteriormente muestran diferencias de país a país; sin embargo, la situación es que las poblaciones involucradas se exponen a una amenaza que impactará su salud y de no revertirse serán una población de adultos con sobrepeso y obesidad. Los adultos con sobrepeso y obesidad enfrentan múltiples problemas como, por ejemplo, disminución de la productividad y altos costos para la salud pública (Katzmarzyk, et al., 2015).

En aras de explicar los resultados que se han descrito antes, Herrera-González, Morera-Castro, Araya, Fonseca-Schmidt, y Vargas (2017), puntualiza que el impacto que estos niveles de sobrepeso y obesidad de la niñez, se debe a aspectos ambientales, específicamente al abuso en el uso de la tecnología en edades temprana, que produce una cultura sedentaria heredable a la vida adulta. También el documento de COMISCA (2014), sobre estrategia para la prevención del sobrepeso y la obesidad en la niñez y adolescencia de Centroamérica y República Dominicana 2014-2025, en forma directa menciona que los contribuyentes responsables de los niveles alarmantes de sobrepeso y obesidad en la niñez son el sedentarismo y los niveles de alimentación inapropiados.

Aunque los hallazgos de este estudio no muestran diferencias significativas entre los niños y las niñas en relación con el IMC, disertaciones en otros países centroamericanos han mostrado una diferencia significativa como, por ejemplo, en el estudio realizado por Reyes, et al. (2018) se presentó un aumento del IMC en niñas con forme el avance de la edad, principalmente durante la adolescencia, lo cual es achacado a un desarrollo puberal más temprano que los niños (Cofré, 2015).

A pesar de la claridad del impacto positivo de actividad física durante la niñez, los hallazgos de este estudio nos muestran niveles de casi un $60 \%$ de sedentarismo; sin embargo, se encuentra por debajo del $70 \%$ reportado por INCAP (2016), lo que mostraría alguna tendencia en el tiempo como para comentar que la niñez actual es más sedentaria de lo que fue en décadas pasadas. En los últimos dos decenios ha habido una disminución constante en la actividad física en la niñez y la adolescencia; al igual que los participantes en este estudio, una gran proporción de niños y niñas no alcanzan las recomendaciones de actividad física (OMS, 2010), sobre todo en los infantes con sobrepeso y obesidad. Con la edad, la actividad física va disminuyendo y, de acuerdo con el sexo, las niñas son más sedentarias que los niños (Raimann y Verdugo, 2012), para el caso de este estudio los datos mostraron cifras equitativas para ambos sexos, sin diferencias significativas en las edades de 11, 12 y 13 años, en relación con la edad y nivel educativo. Sin hacer relación al sexo o la edad, la inactividad física es considerada una epidemia que está afectado a la población mundial, es responsable de la prevalencia de las enfermedades crónicas no transmisibles, como diabetes, hipertensión, algunos tipos de cáncer y enfermedades cardiovasculares (Márquez, Rodríguez y De Abajo, 2006; OMS, 2010).

Las recomendaciones de salud pública para que los niños y niñas realicen por lo menos sesenta minutos de actividad física diaria de intensidad moderada a vigorosa (OMS, 2010), es algo que el estudiantado de esta investigación no cumple, lo que los expone a un mayor riesgo de desarrollar sobrepeso u obesidad (Biddle y Asare, 2011), y muchas otras enfermedades como la diabetes y las enfermedades cardiovasculares (Buchan, 
MHSALUD, ISSN: 1659-097X, 17(1), ENERo-Junio, 2020, PP 1-15

Evert Iraheta, Álvarez Bogantes

Ollis, Thomas y Baker, 2012). Esto es especialmente preocupante cuando hablamos de los niños y niñas que presentan problemas en relación con el peso, ya que la disminución de los niveles de actividad física desde la infancia es más evidente en ellos a través de la adolescencia (Janz, Burns y Levy, 2005). En el caso de las niñas, este estudio no ha demostrado una tendencia a la disminución de la AF en el tiempo, como lo han reportado De Piero, Rodríguez, González y López, (2014), Raimann y Verdugo, (2012) y Reyes, et al. (2018).

En la literatura se ha reportado, que los niños se involucran más en actividades de moderada intensidad que las niñas, independientemente si se cuenta con las condiciones ambientales apropiadas (Pawlowski, Tjørnhøj-Thomsen, Schipperijn y Troelsen, 2014). Por otra parte, el tiempo de juego representa el principal contexto en el que las niñas y los niños tienen la misma oportunidad de ser físicamente activos a diario (Pawlowski, Tjørnhøj-Thomsen, Schipperijn y Troelsen, 2014); sin embargo, se debe considerar que ellas no se involucran espontáneamente en actividades de moderada intensidad, lo que podría ser un factor que interfiere en el desarrollo motor, lo que impide el involucramiento exitoso en actividad física durante el tiempo libre, como lo ha establecido Ridgers, Salmon, Parrish, Stanley y Okely (2012). Esta nueva barrera que limita a muchos niños y niñas para que sean activos físicamente debe ser atendida. Por tanto, es importante, para posibilitar la participación en actividad física durante la infancia, el desarrollar estrategias de intervención desde la visión de los niños y las niñas, con un acercamiento multinivel de la realidad (Buchan, Ollis, Thomas y Baker, 2012).

En relación con la variable autoestima, los alarmantes hallazgos de este estudio encontraron que un 52 $\%$ de los infantes evaluados presentaron niveles bajos de autoestima, los cuales son datos similares a los que encontramos en estudios realizados en Honduras (Lacayo, Check, Vega y Córdoba, 2017) y Nicaragua (Reyes, et al., 2018). Como un elemento indispensable en la búsqueda de elementos que guíen en un mejor entendimiento de los resultados, hay que mencionar que la mayoría de escuelas evaluadas se encuentran ubicadas en zonas controladas por las pandillas, con niveles altos de extorsión y violencia, dando como resultado el desplazamiento y la inmigración de los padres, lo que daña la cohesión familiar y disminuye el apego de los niños a los padres, situación que eventualmente produciría impacto negativo en la autoestima de los niños (Liu, Wu y Ming, 2015).

Una parte importante de los participantes de este estudio pertenecen a un nivel socioeconómico bajo, con familias disfuncionales o poco reforzadoras y están inmersos en ambientes frustrantes en lo concerniente a una formación óptima de la autoestima. La población de referencia niños y niñas podrían mostrar diferencias en la valoración que hacen de sí mismos en función de la variable socioeconómica (Taberno, Serrano y Mérida, 2017). Autores como Marín y Villegas-Robertson (2016), han enfatizado el entorno escolar como un agente básico en la construcción de la autoestima positiva, que facilita el éxito académico, lo que propicia una relación inversa con el fracaso, la violencia escolar, ansiedad y demás indicadores de desajuste emocional.

Como resultado de altos porcentajes de baja autoestima en la muestra evaluada, se hace referencia a los expuesto por Shriver, et al., (2013) que comenta que la población infantil con baja autoestima es susceptible a desórdenes de alimentación, trastornos de la imagen corporal, lo que le impediría desarrollar una vida satisfactoria y exitosa, contribuye al deterioro del bienestar psicológico de los niños (Hojjat, et al., 2016) y a la afectación de los factores psicosociales (Contreras-Valdez, Hernández-Guzmán y Freyre, 2016). Estas dificultades psicológicas son cada vez más comunes en edades tempranas en una sociedad donde la identidad personal y la presión social, continúan siendo un referente para la población en general, independientemente de la edad (Sánchez y Ruiz, 2015; Taberno, Serrano y Mérida, 2017).

De este modo, los niveles de baja autoestima reportados en este estudio, podrían estar relacionados con la alta prevalencia de sobrepeso y obesidad de esta población, al propiciar como consecuencia una afectación de los factores psicosociales (Contreras-Valdez, Hernández-Guzmán y Freyre, 2016). Estas dificultades psicológicas son cada vez más comunes en edades tempranas en una sociedad donde la identidad personal y la presión social, continúan siendo un referente para la población en general, independientemente de la edad (Sánchez y Ruiz 2015; Taberno, Serrano y Mérida, 2017). Aunado a lo anterior Liu, Wu y Ming (2015), hace fines de lucro, desarrollado bajo la iniciativa de acceso abierto 
énfasis acerca de la importancia de la vinculación de los pares y de la buena relación con los padres para el desarrollo y mantenimiento de una autoestima alta. Lo que nos indica que los resultados de esta investigación muestran afectación en estos dos ámbitos.

A la hora de relacionar niveles altos de sobrepeso y obesidad, con los niveles bajos y moderados de actividad física reportados en este estudio, se podría dar como resultado una disminución en la percepción de la autoeficacia, la competencia física y la aceptación física, lo que genera cambios negativos en la autoestima de los niños y niñas. En este punto está clara la asociación e importancia de la actividad física en la construcción y promoción de una autoestima positiva y la autoeficacia en entornos escolares saludables (Batista, Cubo, Honório y Martins, 2016).

Desde el punto de vista de salud pública, los resultados de esta investigación marcan un derrotero para el desarrollo de intervenciones que contemplen el entorno escolar, comunal y familiar, con el fin de propiciar y romper las tendencias de incremento del sobrepeso y la obesidad, el sedentarismo y la baja autoestima a través de estrategias que logren activar a la niñez, de por vida, hacia estilos de vida activos, junto con ambientes que propicien una mejor alimentación en esta población.

\section{Conclusiones}

Los niveles de sobrepeso y obesidad de $23.3 \%$ son alarmantes, pues muestra y comprueba el riesgo latente de esta población, porque supone que en la edad adulta seguirán con la prevalencia de estas condiciones, lo que les llevaría a adquirir enfermedades crónicas no transmisibles, tales como: dislipidemias, hipertensión arterial, diabetes y obesidad, lo que disminuye su calidad de vida y representa una carga para el sistema de salud.

Los resultados obtenidos al medir la actividad física mostraron, con un $53.7 \%$, que existe un nivel bajo y no alcanzan el necesario de actividad física establecido por la Organización Mundial de la Salud, condición que los hace propensos a padecer Enfermedades No Transmisibles (ETN), accidentes cerebro vasculares y algún tipo de cáncer.

Sumado al hecho que los infantes tienen cifras alarmantes de sobrepeso y obesidad con niveles bajos de actividad física, el $52.9 \%$ de la población evaluada presenta una baja autoestima, que podría deberse a una serie de factores psicosociales que interrumpen el desarrollo armónico de su personalidad, lo cual puede propiciar actitudes agresivas, depresión, consumo de sustancias psicoactivas y dificultades en la interacción social.

\section{RECOMENDACIONES}

Los resultados sugieren que es muy importante incrementar el nivel de actividad física y la autoestima, bajar la prevalencia de obesidad y sobrepeso en la población estudiada, esto mediante de una intervención que reúna un equipo multidisciplinario para mejorar los hábitos alimenticios, lograr el incremento de la práctica deportiva y crear estrategias que mejoren el nivel de autoestima.

Crear una Red Nacional de Actividad Física y Calidad de Vida Escolar, con el objetivo de aumentar el nivel de actividad física desde una perspectiva cultural, que reconozca el impacto en la salud y calidad de vida de los infantes, la cual debe estar integrada por universidades, carteras de Estado, organizaciones de sociedad civil e instituciones afines.

Elaborar una Política de Actividad Física y Calidad de Vida Escolar, que garantice un marco jurídico, de manera que establezca acciones para mejorar los hábitos alimenticios, logre el incremento de la práctica deportiva y cree estrategias que aumenten el nivel de autoestima en los escolares salvadoreños. 
MHSALUd, ISSN: 1659-097X, 17(1), ENERO-JUnio, 2020, PP 1-15

Evert Iraheta, Álvarez Bogantes

\section{REFERENCIAS}

Aparco, J. P., Bautista-Olórtegui, C., Astete-Robilliard, L., y Pillaca, J. (2016). Evaluación del estado nutricional, patrones de consumo alimentario y actividad física en escolares del cercano Lima. Revista Peruana de Medicina Experimental y Salud Publica, 33(4) 633-669. http://dx.doi.org/10.17843/rpmesp.2016.334.2545

Araujo, S. V., Cerritos, K. V. y Molina, S. R. (2017). Diseño, implementación y evaluación de un programa psicoterapéutico grupal orientado a fortalecer la autoestima de adolescentes del sexo femenino entre los 13 y 17 años de edad beneficiarias de Fundación Redentor, ubicado en Colonia Escalón, San Salvador. (Tesis de Grado). Departamento de Psicología, Universidad de El Salvador. Recuperado de http://ri.ues.edu.sv/13749/

Batista, M., Cubo, D. S., Honório, S. y Martins, J. (2016). The practice of physical activity related to self-esteem and academical performance in students of basic education. Journal of Human Sport and Exercise, 11(2), 297-310. h ttps://doi.org/10.14198/jhse.2016.112.03

Biddle, S. J. H. y Asare, M. (2011). Physical activity and mental adolescents: a review of reviews. British Journal of Sports Medicine, 45(11), 886-895. http://dx.doi.org/10.1136/bjsports-2011-090185

Buchan, D., Ollis, S., Thomas, N. y Baker, J. (2012). Physical Activity Behaviour: An Overview of Current and Emergent Theoretical Practices. Journal of Obesity, 34, 1-11. http://dx.doi.org/10.1155/2012/546459

Centro de Control y Prevención de Enfermedades [CDC]. (2015). Calculadora del indice de masa corporal para la población infantil y juvenil. Recuperado de https://nccd.cdc.gov/dnpabmi/Calculator.aspx

Cofré, C. (2015). El problema del sobrepeso y la obesidad infantil y sus interacciones con la actividad fisica, el sedentarismo y los habilitos nutricionales en Chile. (Tesis de doctorado) España: Universidad de Granada. http://dx.doi.org/ $10.4067 /$ S0034-98872007000100009

Consejo de Ministros de Salud de Centroamérica y República Dominicana. (COMISCA, 2014). Estrategia para la prevención del sobrepeso y la obesidad en la niñez y adolescencia de Centroamérica y República Dominicana 2014-2025. Recuperado de http://copal.org.ar/wp-content/uploads/2015/06/201406-comisca-estrategia_obe sidad.pdf

Consejo Nacional de Seguridad Alimentaria y Nutricional [CONASAN]. (2016). IV Censo Nacional de talla y I Censo Nacional de peso en escolares de primer grado. Recuperado de http://incap.int/index.php/es/publicaciones/publicaciones-conjuntas-con-otras-instituciones/doc_downlo ad/832-el-salvador-informe-de-resultados-censos-de-talla-y-peso-2017

Contreras-Valdez, J. A., Hernández-Guzmán, L. y Freyre, M. Á. (2016). Body dissatisfaction, self-esteem, and depression in girls with obesity. Revista Mexicana de trastornos alimentarios, 7(1), 24-31. https://doi.org/10.1 016/j.rmta.2016.04.001

Crocker, P. R. E., Bailey, D.A., Faulkner, R.A., Kowalski, K.C. y McGrath, R. (1997). Measuring general levels of physical activity: Preliminary evidence for the Physical Activity Questionnaire for Older Children. Medicine and Science in Sports and Exercise, 29(10), 1344-1349. https://doi.org/10.1097/00005768-199710000-00011

De Piero, A., Rodríguez, E., González, L. y López, A. (2014). Sobrepeso y obesidad en un grupo de escolares españoles. Revista Chilena de Nutrición, 41(3), 264-271 http://dx.doi.org/10.4067/S0717-75182014000300006

Dubon, S. y Aguilar, C. (2018). Análisis de sobrepeso, obesidad, niveles de actividad fisica actividad física y autoestima en la niñez Guatemalteca. [Manuscrito en proceso de publicación].

Gahagan, S. (2016). Overweight and obesity. In: Kliegman RM, Stanton BF, St. Geme JW, Schor NF, eds. Nelson Textbook of Pediatrics. 20th ed. Philadelphia, PA: Elsevier Saunders.

García, C. M. y González-Jurado, J. A. (2017). Impacto de la inactividad física en la mortalidad y los costos económicos por defunciones cardiovasculares: evidencia desde Argentina. Revista Panamericana de Salud Pública, 41(92), 1-8. Recuperado de https://scielosp.org/article/rpsp/2017.v41/e92/es/

Hernández-Sampieri, R., Fernández-Collado, C. y Baptista, M. P. (2010). Metodología de la Investigación. México, D. F.: McGraw-Hill, S. A. 
Herrera-González, E., Morera-Castro, M., Araya, G., Fonseca-Schmidt, H. y Vargas, M. P. (2017). Análisis del estado físico y psicológico de la población escolar del cantón central de Heredia, Costa Rica. MHSalud: Revista en Ciencias del Movimiento Humano y Salud 13(2), 1-17. http://dx.doi.org/10.15359/mhs.13-2.7

Hojjat, S., Fathirezaie, Z., Brand, S., Pühse, U., Holsboer-Trachsler, E., Gerber, M. y Talepasand, S. (2016). Physical activity and self-esteem: testing direct and indirect relationships associated with psychological and physical mechanisms. Neuropsychiatric Disease and Treatment, 12, 2617-2625. https://doi.org/10.2147/NDT.S116811

Instituto de Nutrición de Centroamérica y Panamá [INCAP]. (2016). Situación de la obesidad en Centroamérica y República Dominicana. Unidad de Planificación. Recuperado de http://www.incap.int/sisvan/index.php/es/cooperacion-tecnica-en-la-region/documentos-especializados/d oc_view/279-nota-tecnica-situacion-de-la\%20obesidad-en-centro-america-y-\%20republica-dominicana-2016

Instituto Universitario de Opinión Pública [IUDOP]. (2014). La situación de la seguridad y la justicia 2009-2014. Entre expectativas de cambio, mano dura militar y treguas pandilleras. San Salvador: Instituto Universitario de Opinión Pública (IUDOP). Recuperado de http://www.uca.edu.sv/iudop/wp-content/uploads/libro_la_situa ci\%C3\%B3n_de_la_seguridad.pdf

Janz, K. F., Burns, T. L. y Levy, S. M. (2005). Tracking of Activity and Sedentary Behaviors in Childhood: The Iowa Bone Development Study. American Journal of Preventive Medicine, 29 (3), 171-178. http://dx.doi.org/10.10 16/j.amepre.2005.06.001

Katzmarzyk, P. T., Barreira, T. V., Broyles, S. T., Champagne, C. M., Chaput, J. P., Fogelholm, M., ... \& Church, T. S. (2015). Physical activity, sedentary time, and obesity in an international sample of children. Medicine \& Science in Sports \& Exercise, 47(10), 2062-2069. doi: http://doi.org/10.1249/MSS.0000000000000649

Lacayo, M., Check, E., Vega, N. y Córdova, A. (2018). Análisis de sobrepeso y obesidad, niveles de actividad fisica y autoestima en la niñez de Tegucigalpa, Honduras. [Manscrito en proceso de publicación].

Lawrence, D. (1981). The delevolpment of a self-esteem questionnaire. Brithish Journal Educational Psychology, 51(2), 245-251. https://doi.org/10.1111/j.2044-8279.1981.tb02481.x

Liu, M., Wu, L. y Ming, Q. (2015). How does physical activity intervention improve self-esteem and self-concept in children and adolescents? Evidence from a meta-analysis. PLoS ONE, 1O(8), 1-17. https://doi.org/10.1371/jo urnal.pone. 0134804

Manzano, M. (2016). El Salvador: herencia social de la violencia. Recuperado de: https://www.uls.edu.sv/sitioweb/co mponent $/ \mathrm{k} 2 /$ item/407-el-salvador-herencia-social-de-la-violencia

Marcó, M y de Gracia, M. (2000). Evaluación de la imagen corporal en pre-adolescentes. Girona, España: Universitat de Girona.

Marín, M y Villegas-Robertson, J. M. (2016). Clima, autoestima y violencia escolar en estudiantes de séptimo básico a cuarto medio en una escuela de la comuna de Alto Hospicio, Chile. Revista del Observatorio sobre la violencia y convivencia en la escuela, 2(4), 60-74. Recuperado de https://bit.ly/2D6voae

Márquez, S., Rodríguez, J. y De Abajo, S. (2006). Sedentarismo y salud: efectos beneficiosos de la actividad física. Apunts Educación Física y Deportes, 83, 12-24. Recuperado de https://core.ac.uk/download/pdf/41584471.pdf

Martínez-Gómez, D., Martínez-de-Haro, V., Pozo, T., Welk, G. J., Villagra, A., Calle, M. E., Marcos, A. y Veiga, O. L. (2009). Fiabilidad y validez del cuestionario de actividad física PAQ-A en adolescentes españoles. Revista Española de Salud Pública, 83(3), 427-439. https://doi.org/10.1590/S1135-57272009000300008

Ministerio de Salud de El Salvador [MINSAL]. (2015). Resultados relevantes encuesta nacional de enfermedades crónicas no transmisibles en población adulta de El Salvador. Recuperado de: https://www.salud.gob.sv/archivos/comun icaciones/archivos_comunicados2017/pdf/presentaciones_evento20032017/01-ENECA-ELS-2015.pdf

Ministerio de Salud de El Salvador [MINSAL]. (7 de setiembre de 2016). MINSAL realiza taller de prevención de la obesidad en la niñez. Recuperado de http://www.salud.gob.sv/07-09-2016-minsal-realiza-taller-de-prevencionde-la-obesidad-en-la-ninez/

Organización Mundial de la Salud [OMS]. (2010). Recomendaciones mundiales sobre actividad física para la salud. Geneva: WHO Library Cataloguing-in-Publication, (Completo), 1-58.Recuperado de https://apps.who.int/ iris/bitstream/ 
MHSALUd, ISSN: 1659-097X, 17(1), ENERo-Junio, 2020, PP 1-15

Evert Iraheta, Álvarez Bogantes

handle/10665/44441/9789243599977_spa.pdf;jsessionid=ABB79590DD2ED540283FD8BBFC88B26B? sequence $=1$

Organización Mundial de la Salud [OMS]. (2014). Estadisticas de salud mundiales. Recuperado de: http://apps.who .int/iris/bitstream/10665/112738/1/9789240692671_eng.pdf

Organización Mundial de la Salud [OMS]. (2016). Estrategia mundial sobre régimen alimentario, actividad fisica y salud: Sobrepeso y obesidad infantil. Recuperado de http://www.who.int/dietphysicalactivity/childhood/es/

Organización Panamericana de la Salud [OPS]. (5 de Diciembre de 2017). Análisis de Situación: Enfermedades crónicas no transmisibles. Recuperado de http://www.paho.org/gut/index.php?option=com_content\&view=article\&id $=494:$ banalisis-de-situacion-enfermedades-cronicas-no-transmisibles\&Itemid $=405$

Pawlowski, C. S., Tjørnhøj-Thomsen, T., Schipperijn, J. y Troelsen, J. (2014). Barriers for recess physical activity: A gender specific qualitative focus group exploration. BMC Public Health, 14(1), 1-10. https://doi.org/10.1186 /1471-2458-14-639

Raimann, X. y Verdugo M. F. (2012). Actividad Física en la prevención y tratamiento de la obesidad infantil. Revista Médica Clínica Las Condes, 23(3), 218-225. https://doi.org/10.1016/S0716-8640(12)70304-8

Reyes, R., Otero, E., Pastrán, R., Herrera-Monge, M. y Álvarez, C. (2018). Análisis del sobrepeso, obesidad, niveles de actividad física y autoestima de la niñez de León, Nicaragua. MHSalud: Revista en Ciencias del Movimiento Humano y Salud, 16(1), 1-13. https://doi.org/10.15359/mhs.16-1.4

Ridgers, N. D., Salmon, J., Parrish, A. M., Stanley, R.M. y Okely, A.D. (2012). Physical activity during school recess: A systematic review. American Journal of Preventive Medicine, 43(3), 320-328. http://doi.org/10.1016/j.amep re.2012.05.019

Sánchez, J, J., y Ruiz, A. O. (2015). Relationship between self-esteem and body image in children with obesity. Revista Mexicana de Trastornos Alimentarios, 6(1) 38-44, https://doi.org/10.1016/j.rmta.2015.05.006

Shriver, L. H., Harrist, A. W., Page, M., Hubbs-Tait, L., Moulton, M.y Topham, G. (2013). Differences in body esteem by weight status, gender, and physical activity among young elementary school-aged children. Body Image, 10(1), 78-84. https://doi.org/10.1016/j.bodyim.2012.10.005

Taberno, C., Serrano, A. y Mérida, R. (2017). Estudio comparativo de la autoestima en escolares de diferente nivel socioeconómico Carmen. Psicología Educativa, 23, 9-17. http://dx.doi.org/10.1016/j.pse2017.02.001

Zamani, S. H., Fathirezaie, Z., Brand, S., Pühse, U., Holsboer-Trachsler, E., Gerber, M. y Talepasand, S. (2016). Physical activity and self-esteem: Testing direct and indirect relationships associated with psychological and physical mechanisms. Neuropsychiatric Disease and Treatment, 12, 2617-2625. https://doi.org/10.2147/NDT .S116811

CC BY-NC-ND 